\title{
Symptom Severity and Guideline-Based Treatment Recommendations for Depressed Patients: Implications of DSM-5's Potential Recommendation of the PHQ-9 as the Measure of Choice for Depression Severity
}

\author{
Mark Zimmerman \\ Department of Psychiatry and Human Behavior, Brown Medical School, and Department of Psychiatry, \\ Rhode Island Hospital, Providence, R.I., USA
}

\section{Introduction}

The National Institute for Health and Clinical Excellence (NICE) and the recently revised American Psychiatric Association (APA) guidelines for the treatment of depression both indicate that the severity of depression should be considered when initiating treatment $[1,2]$. The NICE guidelines recommended psychotherapy as the first treatment option for mildly depressed patients. The APA treatment guidelines recommended either psychotherapy and pharmacotherapy as monotherapies for depression of mild or moderate severity, and pharmacotherapy (with or without psychotherapy) for severely depressed patients. If the severity of depression is to influence treatment choice in clinical practice, it is important to have available clinically useful, reliable and valid methods of distinguishing between levels of depression severity.

Many scales have been developed to measure the severity of depression [3]. One of the most frequently used self-administered measures is the 9-item Patient Health Questionnaire (PHQ-9). In describing the initial devel- opment of the PHQ-9, Kroenke et al. [4] recommended cutoff scores corresponding to mild, moderate, moderately severe and severe levels of depression severity, and the validity of these cutoffs was supported by demonstrating differences in health-related quality of life between adjacent severity categories.

In previewing the potential changes in the upcoming fifth edition of the Diagnostic and Statistical Manual, Regier et al. [5] noted that the most important advance of the DSM-5 will be the incorporation of dimensional ratings into the nosology. The DSM-5 Work Group for Depressive Disorders is considering the PHQ-9 as the preferred measure for quantifying the severity of depression. However, there are no studies empirically deriving the most valid cutoffs to demarcate severity gradations. Considering the importance accorded to symptom severity by the APA and NICE treatment guidelines in determining initial treatment efforts, the increasing use of the PHQ-9 in primary care settings, and the potential endorsement in DSM-5 that it be used to measure the severity of depression, it is important to examine the validity of the PHQ-9's severity categorization.

\section{KARGER}

Fax +4161306 1234 E-Mail karger@karger.ch www.karger.com
(C) 2012 S. Karger AG, Basel

0033-3190/12/0816-0329\$38.00/0

Accessible online at:

www.karger.com/pps
Mark Zimmerman, MD

Bayside Medical Center

235 Plain Street

Providence, RI 02905 (USA)

E-Mail mzimmerman@lifespan.org 


\section{Derivation of the PHQ-9 Severity Thresholds}

The PHQ-9 contains 9 items corresponding to the DSM-IV major depressive disorder criteria. The respondent is instructed to rate the symptom items on a 4-point Likert scale indicating how often they have been bothered by the symptom over the past 2 weeks $(0=$ not at all; $1=$ several days; $2=$ more than half the days; $3=$ nearly every day). Total scores on the scale range from 0 to 27. In their initial report on the validity of the PHQ-9, Kroenke et al. [4] indicated that the severity cutoff scores were chosen for the pragmatic reason of making them easier for clinicians to recall. They therefore used 5-point intervals to identify ranges of scores corresponding to severity levels (0-4 indicates no depression; 5-9, mild depression; $10-$ 14, moderate depression; 15-19, moderately severe depression, and 20-27, severe depression). The authors also noted that alternative cutoffs did not increase the association between increasing PHQ-9 severity and indices of construct validity.

To be sure, for all scales measuring the severity of depressive symptoms the thresholds distinguishing patients with mild, moderate and severe depression do not represent well-demarcated lines separating the severity subtypes. As with other areas of psychopathology, the severity of depression better corresponds to a dimensional than a categorical model of classification [6]. However, Kroenke et al. [4] did not consider how the broadness of severity ranges could impact on treatment selection when treatment selection, as described by official guideline recommendations, is guided by symptom severity. If severity distinctions might be used in the selection of treatment, then how broadly or narrowly these categories are defined will have significant clinical implications. It is therefore inappropriate to choose cutoffs to define the severity categories on the basis of their ease of recall.

\section{Does the PHQ-9 Overclassify Depression Severity?}

Kroenke et al. [4] presented the distribution of depressed patients falling into each of the grades of severity, and they found that more than one third of the primary care patients with major depressive disorder were severely depressed according to the PHQ-9, and only $10 \%$ were mildly depressed. We are skeptical that 3 times as many patients in primary care settings have severe depression than mild depression. We are also skeptical that more than one third of depressed primary care patients fall into the severe range. For the past 15 years we have integrated standardized rating scales into our clinical practice as part of the Methods to Improve Diagnostic Assessment and Services (MIDAS) project [7]. To date, we have evaluated more than 1,500 patients who met the DSM-IV criteria for major depressive disorder at the time of the evaluation. Patients were recruited into the MIDAS project without regard to inclusion and exclusion criteria that are typically used in antidepressant effectiveness studies [8]. Thus, similar to the study of Kroenke et al. [4], a minimum level of symptom severity was not required. The patients were rated on the Clinical Global Index of severity [9]. Despite the fact that the majority of patients referred to our practice are from primary care physicians and therapists after they have failed previous treatments, only $20 \%$ of the patients were rated as severely depressed, which is lower than the $34 \%$ rate found by Kroenke et al. [4] according to the PHQ-9.

There is direct evidence that the PHQ-9 overestimates severity when classifying grades of severity compared to other measures. Studies comparing the PHQ-9 to the depression subscale of the Hospital Anxiety and Depression Scale (HADS-D) [10] have found that more patients are classified as severely depressed on the PHQ-9. Hansson et al. [11] administered the PHQ-9 and HADS-D to 737 patients presenting with depressive symptoms in 5 primary care and 5 psychiatric centers in Sweden. More than twice as many patients were classified as severely depressed on the PHQ-9 than the HADS-D (23.9 vs. $10.0 \%$ ). Cameron et al. [12] compared the distribution of PHQ-9 and HADS-D grades of severity in primary care patients referred for mental health treatment in 32 practices in Scotland. The diagnostic composition of the sample was not specified. They found that approximately twice as many patients were in the severe range on the PHQ-9 than the HADS-D (19 vs. $10 \%)$. At the other end of the spectrum, on the HADS-D, more patients were in the nondepressed (33 vs. $8 \%$ ) and mildly depressed (30 vs. $18 \%)$ range.

In another study of 267 primary care patients diagnosed with depression, Cameron et al. [13] compared severity classification on the PHQ-9, Hamilton Depression Rating Scale (HAMD) [14], HADS-D and Beck Depression Inventory [15]. They found that the PHQ-9 and Beck Depression Inventory classified patients in a severer category compared to the HAMD and HADS-D, though the authors did not present information on the percentage of patients classified as severely depressed by each measure.

One study examined the validity of PHQ-9 severity categorization in psychiatric patients. Zimmerman et al. [16] evaluated 245 outpatients with DSM-IV major de- 
pressive disorder who presented for treatment or who were under ongoing treatment and had their medication changed due to lack of efficacy. The patients completed the PHQ-9 and were evaluated with the 17-item HAMD at baseline. Four times as many patients scored in the mild range on the HAMD than the PHQ-9 (29.9 vs. 7.4\%). Conversely, significantly fewer patients were classified as severely depressed on the HAMD compared to the PHQ9 (25.0 vs. $38.9 \%)$. If the patients in the moderately severe range on the PHQ-9 were combined with the severe group, then the prevalence of severe depression was almost 3 times higher on the PHQ-9 (69.3 vs. 25.0\%). When the PHQ-9's moderately severe group was subdivided into moderate depression (scores of 15, 16 and 17) and severe depression (scores of 18 and 19), then the rate of severe depression was twice as high on the PHQ-9 compared to the HAMD (50.4\% vs. $25.0 \%)$.

\section{Implications of DSM-5's Adoption of a Measure That Overclassifies Depression Severity}

The results of these studies raise concerns regarding the validity of the PHQ-9 thresholds for symptom severity categorization. In contrast to the diagnostic properties of the PHQ-9, which have been extensively studied [17], the validity of the PHQ-9 in demarcating levels of depression has received little attention. Before the PHQ-9 is recommended in DSM-5 as the preferred measure of the severity of depression, much more research is needed to determine the most valid cutoffs for severity categories. The importance of establishing valid thresholds for grades of severity is underscored by the recommendations in official treatment guidelines for depression to consider severity when selecting a patient's initial treatment modality $[1,2]$. If one band of severity is overinclusive, another is necessarily underinclusive, and treatment allocation will be accordingly biased.

Standardized scales are not typically used in clinical practice $[18,19]$. In the past few years there have been increasing calls for the utilization of such measures, and it is likely that self-report scales are more likely to be used than clinician-rated scales such as the HAMD. We anticipate future studies examining how well clinicians adhere to official treatment guidelines, and the impact of baseline severity on initial treatment selection is a potential topic of interest. The results of several studies suggest that compared to other depression severity scales the PHQ-9 is overinclusive in classifying patients with severe depression, and correspondingly underinclusive in clas- sifying patients with mild depression. The adoption of such a measure in the DSM-5, which broadly defines the severe group, could result in fewer psychotherapy referrals and greater reliance on medication as a first-line treatment option.

If clinicians are to follow the recommendations of treatment guidelines regarding the impact of severity on initial treatment selection, then it is important that the grades of severity based on cutoff scores on a symptom scale be well established. A macroanalysis of the PHQ-9, of the type described by Tomba and Bech [20], has not yet been conducted. While I agree with the goal of the DSM5 to adopt quantitative, dimensional measures of depression in clinical practice, I would caution against the use of scales to guide treatment selection until the thresholds to define severity ranges have been well established empirically. There has been a long-standing concern about diagnostic thresholds for psychiatric disorders, and the DSM-III and its successors have been accused of overexpanding the definition of mental illness thereby resulting in inflated community prevalence estimates of psychiatric disorders [21]. The consequence of false-positive diagnoses is the overprescribing of treatment, particularly psychotropic medication [22]. The adoption by the DSM5 of severity measures that overclassify severity, in concert with APA treatment guidelines that link increased severity to recommendations to prescribe medication, is likely to further fuel the trend towards overprescribing. Moreover, in the context of this concern about possible overreliance on medication to treat depression, the adoption of the PHQ-9, which is copyrighted by a drug company, will be fodder for critics who contend that the pharmaceutical industry has influenced the content of the DSM and official treatment guidelines [23].

In conclusion, in the absence of sufficient empirical data establishing cutoffs for severity categories, it is premature for the DSM-5 to recommend that the PHQ-9 be used to measure the severity of depression. Moreover, I am concerned that embracing the PHQ-9 as the preferred measure of depression severity will result in accusations that the APA endorsed the measure in order to favor pharmacological over psychotherapeutic approaches towards depression treatment.

\section{Disclosure Statement}

The author has no conflict of interest to declare. 


\section{References}

1 American Psychiatric Association: Practice Guideline for the Treatment of Patients with Major Depressive Disorder, ed 3. Washington, American Psychiatric Association, 2010.

2 National Collaborating Centre for Mental Health: Depression: the treatment and management of depression in adults. NICE Clinical Guideline 90. London, National Institute for Health and Clinical Excellence, 2009, p 64.

3 Nezu A, Ronan G, Meadows E, McClure K: Practitioner's Guide to Empirically Based Measures of Depression. New York, Kluwer Academic/Plenum Publishers, 2000.

4 Kroenke K, Spitzer R, Williams J: The PHQ9. Validity of a brief depression severity measure. J Gen Intern Med 2001;16:606-613.

5 Regier DA, Narrow WE, Kuhl EA, Kupfer DJ: The conceptual development of DSM-V. Am J Psychiatry 2009;166:645-650.

-6 Ruscio J, Zimmerman M, McGlinchey JB, Chelminski I, Young D: Diagnosing major depressive disorder. XI. A taxometric investigation of the structure underlying DSM-IV symptoms. J Nerv Ment Dis 2007;195:10-19.

7 Zimmerman M: Integrating the assessment methods of researchers in routine clinical practice: the Rhode Island Methods to Improve Dignostic Assessment and Services (MIDAS) project; in First MB (ed): Annual Review of Psychiatry. Washington, American Psychiatric Press, 2003, pp 29-34.
8 Zimmerman M, Posternak MA, Chelminski I: Symptom severity and exclusion from antidepressant efficacy trials. J Clin Psychopharmacol 2002;22:610-614.

9 Guy W: ECDEU Assessment Manual for Psychopharmacology. Rockville, National Institute of Mental Health, 1976.

10 Zigmond AS, Snaith RP: The hospital anxiety and depression scale. Acta Psychiatr Scand 1983;67:361-370.

11 Hansson M, Chotai J, Nordstom A, Bodlund O: Comparison of two self-rating scales to detect depression: HADS and PHQ-9. Br J Gen Pract 2009;59:e283-e288.

12 Cameron IM, Crawford JR, Lawton K, Reid IC: Psychometric comparison of PHQ-9 and HADS for measuring depression severity in primary care. Br J Gen Pract 2008;58:32-36.

13 Cameron IM, Cardy A, Crawford JR, du Toit SW, Hay S, Lawton K, et al: Measuring depression severity in general practice: discriminatory performance of the PHQ-9, HADS-D, and BID-II. Br J Gen Pract 2011; 61:e419-e426.

14 Hamilton M: A rating scale for depression. Neurol Neurosurg Psychiatry 1960;23:5662.

15 Beck AT, Ward CH, Mendelson M, Mock J, Erbaugh J: An inventory for measuring depression. Arch Gen Psychiatry 1961;4:561571.
16 Zimmerman M, Martinez J, Friedman M, Attiullah N, Boerescu D, Toba C: How can we use depression severity to guide treatment selection when measures of depression categorize patients differently? J Clin Psychiatry, in press.

-17 Manea L, Gilbody S, McMillan D: Optimal cut-off score for diagnosing depression with the Patient Health Questionnaire (PHQ-9): a meta-analysis. CMAJ 2012;184:E191-E196.

18 Zimmerman M, McGlinchey JB: Why don't psychiatrists use scales to measure outcome when treating depressed patients? J Clin Psychiatry 2008;69:1916-1919.

19 Gilbody SM, House AO, Sheldon TA: Psychiatrists in the UK do not use outcomes measures. National survey. Br J Psychiatry 2002; 180:101-103.

20 Tomba E, Bech P: Clinimetrics and clinical psychometrics: macro- and micro-analysis. Psychother Psychosom 2012;81:333-343.

21 Frances A: Problems in defining clinical significance in epidemiological studies. Arch Gen Psychiatry 1998;55:119.

2 Batstra L, Frances A: Holding the line against diagnostic inflation in psychiatry. Psychother Psychosom 2012;81:5-10.

23 Cosgrove L, Shaughnessy AF, Wheeler EE, Austad KE, Kirsch I, Bursztajn HJ: The American Psychiatric Association's guideline for major depressive disorder: A commentary. Psychother Psychosom 2012;81: 186-188. 